\title{
Performance and Calibration of the ATLAS Jet Trigger
}

\author{
J. Machado Miguéns* on behalf of the ATLAS Collaboration \\ LIP-Lisbon, Portugal \\ E-mail: jmiguens@cern.ch
}

ATLAS has been successfully collecting $7 \mathrm{TeV} p p$ collision data from the LHC since 2010, at a rate that will ultimately reach $40 \mathrm{MHz}$ for nominal LHC conditions. The ATLAS trigger system, organized in three levels, handles this enormous data flow, providing efficient rejection by reconstructing and selecting high transverse momentum objects and reducing the rate to $\approx 300-400$ $\mathrm{Hz}$. High performance of the jet trigger is essential for a wide variety of physics analysis in ATLAS, ranging from QCD studies to SUSY and Higgs searches. In this contribution, the challenges, implementation and performance of the ATLAS jet trigger are presented based on $7 \mathrm{TeV}$ collision data. In addition, possible future improvements of the system are discussed.

XXIst International Europhysics Conference on High Energy Physics

21-27 July 2011

Grenoble, Rhône-Alpes France

*Work financed in part by FCT, Portugal, through the grant SFRH/BD/69173/2010, co-financed by QREN/COMPETE of European Union ERDF fund. 


\section{Introduction}

ATLAS [1] is a general-purpose detector designed for the LHC that has been, since March $30^{\text {th }}$ 2010, collecting $7 \mathrm{TeV}$ collision data. As the LHC increased the delivered instantaneous luminosity, the proton bunch spacing ultimately reached $50 \mathrm{~ns}$, with up to $13 \mathrm{pp}$ collisions occurring per bunch crossing. This poses stringent requirements on the trigger system [1], which has to reject events online, maintaining an unbiased efficiency for rare signals. It is divided in three levels, that identify high $p_{\mathrm{T}}$ objects, such as leptons or jets, and reduce the rate to $\approx 300-400 \mathrm{~Hz}$. The first level (L1) is hardware-based, while the High Level Trigger (HLT), which comprises the second level (L2) and the third level (Event Filter or EF), is based on software running in commercial computers.

\section{Implementation and performance of the ATLAS jet trigger}

In an environment where jets are the most commonly produced objects, high performance of the jet trigger [2] is fundamental to achieve the physics goals of ATLAS. With several inclusive single jet and multijet, combination and topological triggers available in ATLAS, both in the central $(|\eta|<3.2)$ and forward regions of the detector $(3.2<|\eta|<4.9)$, events triggered on jets can be used in a variety of physics analysis, ranging from QCD to SUSY and Higgs searches.

At L1, the jet trigger is implemented in custom made electronic modules operating with coarse granularity of $0.8 \times 0.8$ in $\eta \times \phi$. A sliding window algorithm, which identifies a local $E_{\mathrm{T}}$ maximum in calorimeter trigger towers, is used and if $E_{\mathrm{T}}$ is above the threshold the event is accepted. L2 accesses calorimeter cells, operating with full granularity. It uses a L1 seed as starting point and reconstructs jets in a small region around it (Region of Interest or RoI) using a simplified version of the cone algorithm with a distance parameter of $R=0.4$ in $(\eta, \phi)$ space. The EF has recently undergone major changes that have been instrumental in improving the jet trigger. It currently reads out the whole calorimeter for events that passed L2, which provides more flexibility for the jet trigger and increases its acceptance. The anti- $\mathrm{k}_{\mathrm{t}}$ jet algorithm [4], with $R=0.4$ and $R=1.0$, is currently running online, although other algorithms can be used. Using this algorithm, which is theoretically superior than the previously used cone algorithm and is also the one used offline as default, has improved the EF jet resolution with respect to offline and, thus, increased rejection. Topological clusters [2] serve as input to jet reconstruction at EF, which has increased the stability of EF under pile-up. Jet energies in the trigger are measured at the electromagnetic scale ${ }^{1}$.

The efficiency of several jet trigger chains, depicted in figure 1, was tested with respect to offline jets using data collected in 2011 [3]. Both the central and the forward regions of the detector were analyzed in a wide jet $E_{\mathrm{T}}$ range. Results show the jet trigger is behaving well and efficiently rejecting events, revealing only a weak dependance on pile-up.

Future developments that will contribute to further improve the performance of the jet trigger include: removing spurious jets at L2, appearing due to detector effects or non-collision backgrounds; suppressing pile-up noise at L2 and the EF; using L2 and EF jets calibrated at the hadronic scale, improving the L2 and EF resolution relative to offline; a better integration between L1 speed and

\footnotetext{
${ }^{1}$ The electromagnetic scale is the basic calorimeter signal scale for the ATLAS calorimeters. It gives the correct scale for the energy deposited in electromagnetic showers, while it does not correct for the lower hadron shower response nor for energy losses in dead material.
} 
L2 flexibility.
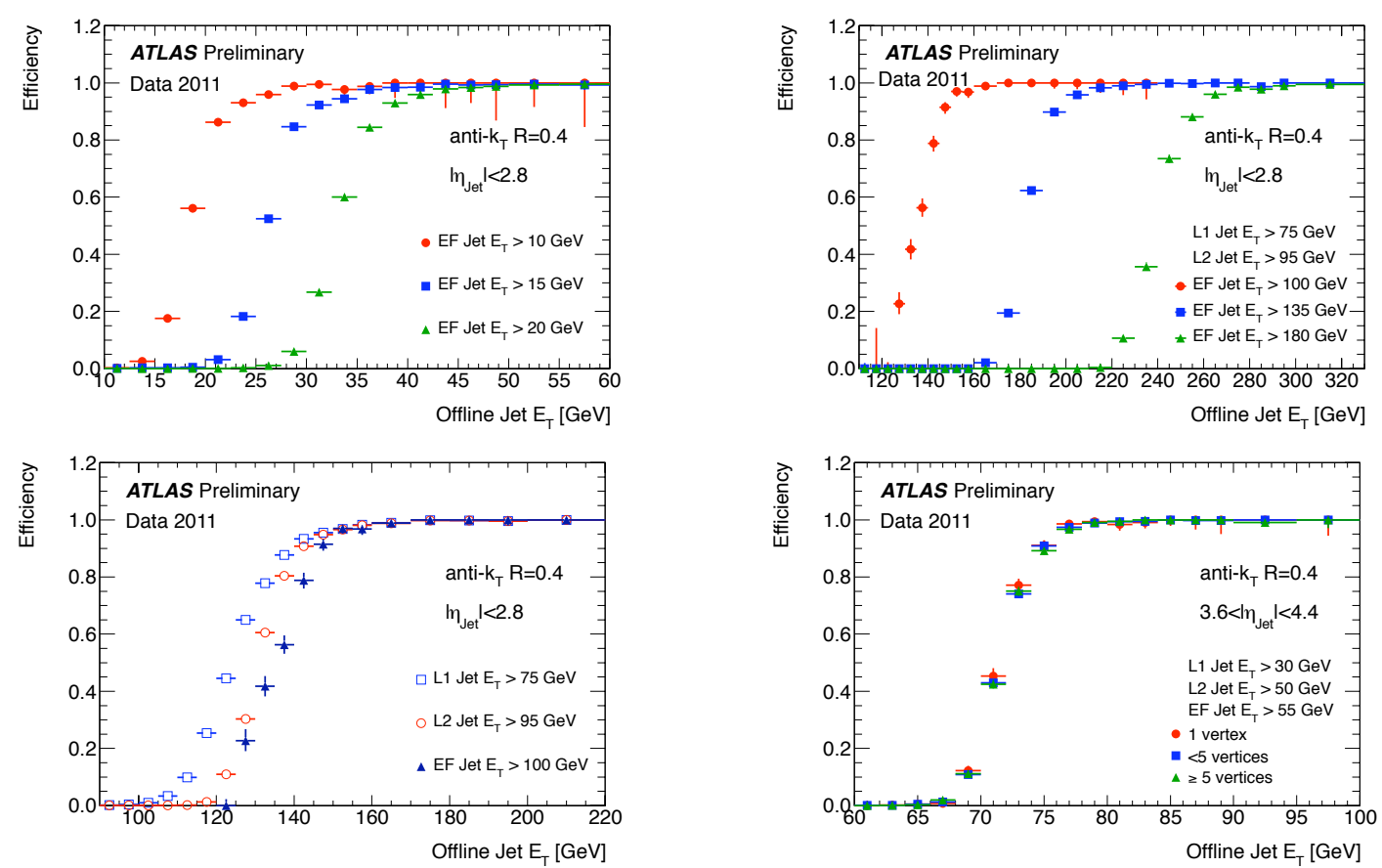

Figure 1: Efficiency for offline anti- $\mathrm{k}_{\mathrm{t}}$ jets with $R=0.4$, as a function of the calibrated jet $E_{\mathrm{T}}$, to satisfy different jet triggers. Top: EF inclusive jet trigger for three choices of low $E_{\mathrm{T}}$ thresholds (left) and three choices of high $E_{\mathrm{T}}$ thresholds (right), for jets with central rapidities. Bottom left: L1, L2 and EF inclusive jet trigger for a single trigger chain, for jets with central rapidities. Bottom right: EF inclusive jet trigger binned in the number of reconstructed $p p$ interactions, for jets with forward rapidities.

\section{Summary and Conclusions}

The ATLAS jet trigger is implemented in three levels, that identify and select events with high $E_{\mathrm{T}}$ jets, both in the central and forward region of the detector. The performance of the ATLAS jet trigger was evaluated with data taken in 2011 and results show it is efficiently selecting events, even under high pile-up conditions. Studies are ongoing to further improve the performance of the ATLAS jet trigger.

\section{References}

[1] ATLAS Collaboration, The ATLAS experiment at the CERN Large Hadron Collider, JINST 3 (2008) S08003.

[2] ATLAS Collaboration, Expected performance of the ATLAS experiment: detector, trigger and physics, CERN-OPEN-2008-020, [arXiv: 0901.0512$].$

[3] ATLAS Collaboration, Inclusive Jet Trigger Efficiencies for the Early 2011 Data, ATLAS-COM-DAQ-2011-031.

[4] M. Cacciari, G. Salam, G. Soyez, The anti-kt jet clustering algorithm, JHEP 0804 (2008) 063, [arXiv:0802.1189]. 